\title{
Unexplained fever in neonates may be associated with hepatitis $B$ vaccine
}

Nehama Linder, Meirav Raz, Lea Sirota, Brian Reichman, Dan Lubin, Jacob Kuint, Avner Herman Cohen, Asher Barzilai

\begin{abstract}
Aim-To investigate whether hepatitis B vaccination has increased the number of cases of unexplained neonatal fever.

Method-The files of all infants born from 1 January 1991 to 31 December 1992, in whom a diagnosis of "injected antibiotic" or "disease of temperature regulation" was recorded, were reviewed. Those who had unexplained fever of $38^{\circ} \mathrm{C}$ or higher during the first three days of life were divided into two groups: infants who did not receive the hepatitis $B$ vaccine (1991) and infants who did (1992).

Results-In 1992 the incidence of unexplained fever in hepatitis $B$ vaccinated neonates was significantly higher than in the 1991 group of pre-vaccination neonates (35 out of $5819(0.6 \%)$ vs 14 out of 5010 neonates $(0.28 \%)$ respectively, $\mathrm{p}=\mathbf{0 . 0 1 3 )}$.

Conclusions-The increase in the number of cases of unexplained neonatal fever seems to be associated with the introduction of routine hepatitis $B$ vaccination on the first day of life. The possibility that an excess number of neonates will undergo unnecessary procedures and treatment to diagnose unexplained fever justifies planning a controlled study to determine whether these preliminary findings point to a significant problem.
\end{abstract}

(Arch Dis Child Fetal Neonatal Ed 1999;81:F206-F207)

Department of Neonatology Schneider Children's

Medical Center of

Israel

14 Kaplan St

Petah Tikva 49202

Israel

N Linder

L Sirota

Department of

Neonatology

Chaim Sheba Medical

Center

Tel Hashomer

and the Sackler School

of Medicine

Tel Aviv University

M Raz

B Reichman

D Lubin

J Kuint

Department of

Paediatric Infectious

Diseases

A H Cohen

A Barzilai

Correspondence to:

Dr N Linder.

Accepted 17 May 1999 Table 1 Comparison of infants with neonatal fever before (1991) and after (1992) introduction of routine hepatitis $B$ immunisation

\begin{tabular}{lrrl}
\hline & $\begin{array}{c}1991 \\
\text { group }\end{array}$ & $\begin{array}{c}1992 \\
\text { group }\end{array}$ & p Value \\
\hline Total infants & 5010 & 5819 & \\
Neonatal fever above $37.5^{\circ} \mathrm{C}$ & 27 & 68 & 0.001 \\
Neonatal fever above $38.0^{\circ} \mathrm{C}$ & 27 & 50 & 0.05 \\
Explained neonatal fever & 13 & 15 & NS \\
Unexplained neonatal fever & 14 & 35 & 0.013 \\
\hline
\end{tabular}

Methods

We reviewed the charts of all full term infants (37 weeks of gestation or more and birthweight $\geqslant 2.5 \mathrm{~kg}$ ) born at the Chaim Sheba Medical Center, Israel, from 1 January 1991 to 31 December 1992, who had a discharge diagnosis of "temperature regulation disease" or who had received intravenous antibiotics as an inpatient. In our department temperature is measured using a digital rectal thermometer (IIVAC 281 model 811, San Diego, CA) in all instances of suspected fever.

"Unexplained" neonatal fever was defined as a temperature of $\geqslant 38^{\circ} \mathrm{C}$ during the first three days of life in the absence of sepsis (positive blood and/or cerebrospinal fluid cultures), dehydration (loss of at least $10 \%$ of birthweight), maternal fever ( $\geqslant 38^{\circ} \mathrm{C}$ at delivery), or respiratory distress (oxygen required for $>4$ hours after birth). Chart entries concerning these specific diagnostic details for infants not vaccinated with hepatitis B, born during 1991, were compared with entries for infants born during 1992, who were vaccinated against hepatitis B. No other changes in nursery staff or routine policies had occurred during the two year period.

\section{Results}

Twenty seven out of 5010 full term infants born in 1991 and 68 out of 5819 born in 1992 had had a diagnosis of neonatal fever. Initial review of the 1992 group yielded 18 infants with fever $<38^{\circ} \mathrm{C}$ who were excluded from the final analysis (table 1). Further review of case files revealed identifiable causes of fever (sepsis, dehydration, maternal fever, respiratory distress) in 13 infants in the 1991 and 15 infants in the 1992 groups, respectively. These infants were excluded from analysis (table 1).

Among the remaining infants with unexplained fever occurring within three days of birth, an increase of more than $100 \%$ was noted between 1991, when hepatitis B vaccine was not administered (0.28\%), and 1992, when hepatitis was given routinely in the first day of life to all neonates $(0.6 \%)(p=0.013)$ (table 1).

All charts of infants with unexplained fever 
from 0 to 7.3 hours. ${ }^{12}$ A lower rate of adverse events was reported in infants and children than in adults. ${ }^{1}$ In neonates the most common susbequent event was fever $\left(37.5-39.0^{\circ} \mathrm{C}\right)$ and was the most common serious sign reported by the Vaccine Adverse Event Reporting System (VAERS). ${ }^{2}$ Their median reported time from vaccination to onset of fever was 1 day and mean maximum temperature was $38.9^{\circ} \mathrm{C}$.

We reviewed the charts of infants with the diagnosis of temperature regulation disease, and of those who received intravenous antibiotics, to identify all infants with neonatal fever. The percentage of infants with unexplained fever during the first three days of life was significantly higher in $1992(0.6 \%)$ when hepatitis $B$ vaccine was given routinely on the first day of life to all neonates compared with 1991 $(0.28 \%)$ when it was not given. The $0.32 \%$ difference is compatible with the 0 to $7.3 \%$ reported rate of febrile reaction to the vaccine. ${ }^{1}{ }^{24-9}$ Notably, had we included infants with a temperature above $37.5^{\circ} \mathrm{C}$, the significance would have been greater $(p<0.001)$. Furthermore, infants born to mothers with maternal fever were excluded from our analysis even though some should probably have been classified as having unexplained neonatal fever.

The more than twofold increase in the percentage of infants with unexplained fever was not associated with the increased rate of enteroviral illnesses in 1992. The monthly distribution of cases of unexplained neonatal fever was stable, except for November 1992, when eight cases were noted. No other changes in our nursery staff or routine policies were introduced during 1992.

In the report by VAERS 24 neonates had severe neonatal events after hepatitis $B$ vaccination; fever was the most common serious neonatal event and was reported in 13 (54\%) neonates. The 13 neonates with fever reported by VAERS were admitted for a median of three days and 10 underwent evaluation for sepsis. ${ }^{2}$ In our study, all 35 neonates underwent a full sepsis evaluation, intravenous antibiotic treatment, and prolonged hospital stay.

Although Israel has a low incidence of hepatitis B carriers among the general Jewish Israeli population $0.5-0.6 \%,{ }^{10}$ the Ministry of Health decided to provide active immunisation for every neonate on the first day of life, mainly because of the large scale immigration from countries in which the virus is hyperendemic. It was expected that since the rate of transmission of anti-hepatitis B antibodies from Israeli mothers to offspring is only $23 \%$, there would be a good antibody response to an early first immunisation. ${ }^{10} 11$

In conclusion, we found that an increased incidence of unexplained neonatal fever, which resulted in evaluation for sepsis, administration of intravenous antibiotics, and prolonged hospital stay, may be associated with vaccination against hepatitis B on the first day of life. Although our data are significant, our numbers are small, therefore a larger controlled trial is justified to determine if the benefit conferred by universal vaccination of neonates against hepatitis B is outweighed by the risks and costs of unnecessary diagnostic procedures and treatments.

1 Andre FE. Summary of safety and efficacy data on a yeastderived hepatitis B vaccine. Am F Med 1989;87(Suppl 3A): $14 \mathrm{~S}-20 \mathrm{~S}$

2 Manette TN, David M, Davis BS, Ellenberg S. Recombinant hepatitis B vaccination of neonates and infants: Emerging safety data from the Vaccine Adverse Event Reporting System. Pediatr Infect Dis f 1996;15:771-6.

3 Centers for Disease Control. Hepatitis B virus: A comprehensive strategy for eliminating transmission in the United States through universal childhood vaccination: recommendations of the Immunization Practices Advisory 1991:40(Sup (ACIP). Morbid

4 Stevens CE, Taylor PE, Tong MJ, Toy PT, Vyas GN, Nair PV. Yeast recombinant hepatitis B vaccine: Efficacy with hepatitis B immune globulin in prevention of hepatitis B virus transmission. $\mathscr{F} A M A$ 1987;257:2612-16.

5 Polakoff S, Vandervelde EM. Immunization of neonatal at high risk of hepatitis B in England and Wales: National surveillance. BMF 1988;297:249-53.

6 Gallo IA, Petrosillo N, Celleti S. Results of neonatal vaccination against hepatitis $\mathrm{B}$ in Frosinone. Ann Ig vaccination again

7 del Canho R, Grosheide PM, Voogd M. Immunogenicity of 20 micrograms of recombinant DNA hepatitis B vaccine in healthy neonates: A comparison of three different vaccination schemes. F Med Virol 1993;41:30-4.

8 Lee CY, Huang LM, Chang MH, Hsu CY, Wu SJ, Sung JL, Safary A. The protective efficacy of recombinant hepatitis $\mathrm{B}$ vaccine in newborn infants of $\mathrm{HBeAg}$ positive hepatitis B surface antigen carrier mother. Pediatr Infect Dis $f$ 1991;10:299-303.

9 West DJ, Calandra GB, Ellis RW. Vaccination of infants and children against hepatitis B. Pediatr Clin North Am 1990;37:585-601.

10 Bogomolski-Yahalomm V, Granot E, Linder N, Adler R, Korman S, Manny N. Prevalence of HBsAg carriers in native and immigrant pregnant female population in Israel and passive/active vaccination against $\mathrm{HBV}$ of newborns at risk. F Med Virol 1991;34:217-32.

11 Isacson M, Halevy J, Eidelman Al, Rudensky B, Tadmor OP, Slater P. Prevalence of HBsAg carriers in pregnant women in Jerusalem: Risk for horizontal transmission to family members. Israel f Med Sci 1994;30:368-70. 